\title{
Alveolar macrophages from patients with tuberculosis exhibit reduced capacity of restricting growth of Mycobacterium tuberculosis: a pilot study of vitamin D stimulation in vitro
}

\author{
Persson H. Lennart ${ }^{1 *}$, Eklund $\mathrm{D}^{2}$, Larsson $\mathrm{M}^{4}$, Welin $\mathrm{A}^{2,3}$, Paues $\mathrm{J}^{5}$, Idh $\mathrm{J}^{2}$, Fransson SG ${ }^{6}$, Stendahl $\mathrm{O}^{2}$, Lerm $\mathrm{M}^{2}$ and Schön $\mathrm{T}^{7}$ \\ ${ }^{*}$ Correspondence: Lennart.Persson@liu.se \\ 'Division of Pulmonary Medicine, Department of Medical and Health Sciences, Faculty of Health Sciences, Linköping University, \\ Department of Respiratory Medicine UHL, Center of Surgery and Oncology, County Council of Östergötland, Linköping, Sweden. \\ ${ }^{2}$ Division of Microbiology and Molecular Medicine, Department of Clinical and Experimental Medicine, Faculty of Health Sciences, \\ Linköping University, Linköping, Sweden. \\ ${ }^{3}$ Phagocyte Research Laboratory, Department of Rheumatology and Inflammation Research, Sahlgrenska Academy, \\ University of Gothenburg, Gothenburg, Sweden. \\ ${ }^{4}$ Division of Clinical Microbiology, Department of Clinical and Experimental Medicine, Lab1, level 10, Faculty of Health Sciences, \\ Linköping University, Linköping, Sweden. \\ ${ }^{5}$ Division of Infectious Diseases, Department of Medical and Health Sciences, University of Linköping, Linköping, Sweden. \\ ${ }^{6}$ Division of Radiology, Department of Medical and Health Sciences, University of Linköping, Linköping, Sweden. \\ ${ }^{7}$ Department of Clinical Microbiology and Infectious diseases, Kalmar County Hospital, Kalmar, Sweden.
}

\begin{abstract}
Background: The role of vitamin D supplementation as adjuvant treatment of tuberculosis (TB) has lately attracted increasing interest. Our aim was to investigate the capacity of alveolar macrophages (AMs) from patients with or without exposure to TB to control intracellular growth of virulent Mycobacterium tuberculosis (Mtb).

Methods: AMs were freshly harvested from the bronchoalveolar lavage fluid of 7 patients with a history of TB (4 patients with previous TB and 3 patients with current TB) and 4 non-TB subjects. The H37Rv strain, genetically modified to express Vibrio harveyi luciferase, was used to determine the growth of Mtb by luminometry in the AMs from study subjects. Cytokine levels in culture supernatants were determined using a flow cytometry-based bead array technique.

Results: AMs from patients with a TB history were less efficient in restricting Mtb growth. Stimulation with $100 \mathrm{nM} 1$, 25 -dihydroxyvitamin $\mathrm{D}\left(1,25 \mathrm{D}_{3}\right)$ did not significantly influence the capacity of AMs from any study subjects to control the infection. Out of the cytokines evaluated (TNF- $\alpha$, IL-1 $\beta$, IL-10 and IL-12p40) only TNF- $\alpha$ demonstrated detectable levels in culture supernatants, but did not respond to stimulation with $1,25 \mathrm{D}_{3}$.

Conclusions: We conclude that AMs of TB-patients show reduced ability to control mycobacterial growth in vitro, and, that AMs in this pilot study do no respond to $1,25 \mathrm{D}_{3}$-stimulation. The former observation supports the concept that innate immunity is crucial for the control of TB infection.
\end{abstract}

Keywords: Alveolar macrophages, bronchoalveolar lavage, cytokines, H37Rv, tuberculosis

\section{Introduction}

Infection with Mycobacterium tuberculosis (Mtb), the causative agent of tuberculosis (TB) has several outcomes in the host $[1,2]$. During the last decades, it has become increasingly clear that some individuals have a highly effective innate immune response against Mtb, which will kill the bacteria upon exposure even before it disseminates and induces cellmediated immunity $[1,3]$. While the vast majority among those who develop infection exhibits latent disease, small children or immune-compromised individuals may develop progressive infection [2]. Vitamin D is known to induce anti-mycobacterial effects through an up-regulation of the anti-microbial peptide cathelicidin in experimental models including mononuclear cells from healthy donors [4-7]. However, the relative importance of vitamin $D$ and the mechanisms at the site of infection has not been confirmed in clinical trials [8], other than in subgroups of patients with polymorphisms in the vitamin D receptor [9].

Much of the knowledge of the innate and adaptive immune mechanisms that are crucial for the control of TB is derived from animal models or from in vitro models based on cell lines or monocyte-derived macrophages obtained from the blood of healthy volunteers $[1,2]$. Although it is known from epidemiological studies that the innate response to Mtb may clear the infection in some individuals, there are few, if any, studies investigating this effect at the cellular level of human alveolar macrophages (AMs) freshly harvested from bronchoalveolar lavage fluid (BALF) from TB patients.

In the present study we investigated the capacity of AMs, obtained from patients with previous or current TB, to inhibit growth of virulent Mtb using a newly developed luminescensebased model for detection of intracellular Mtb growth. In addition, the study evaluated the effects of exogenous 
Lennart et al. Microbiology Discovery 2013,

Table 1. Characteristics of the population studied. Data are presented as means \pm SD. BALF: bronchoalveolar lavage fluid. Significant difference from "non-TB" subjects is indicated; ${ }^{\star} \mathbf{p}<0.05$ (Students t-test, unpaired analysis).

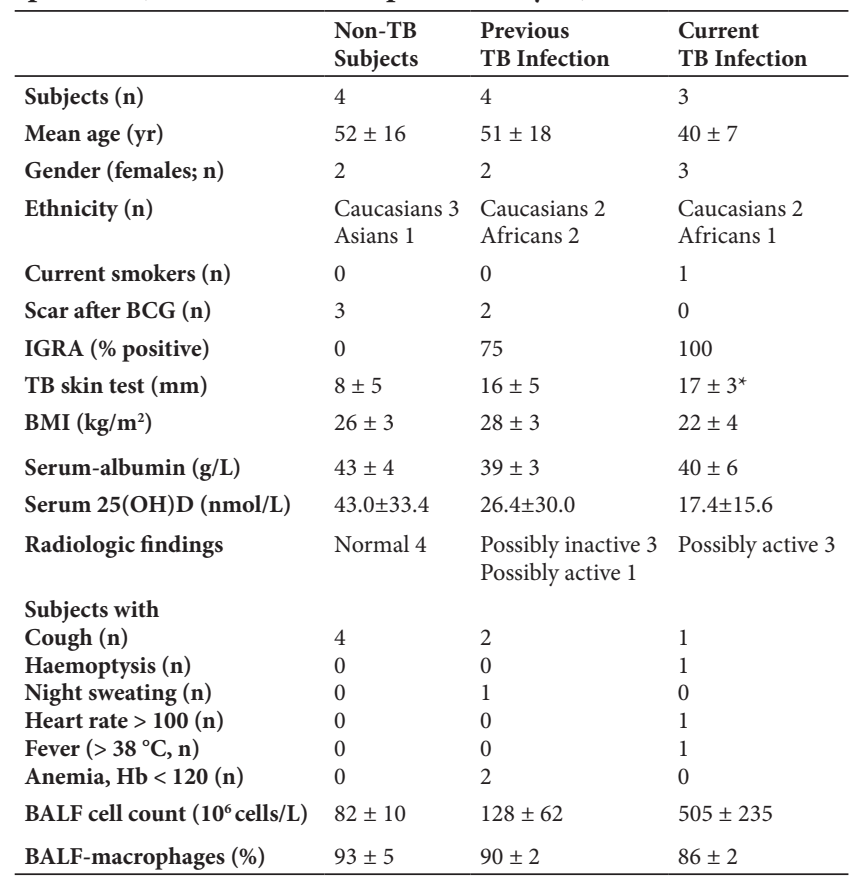

active vitamin $D$ (hereafter referred to as $1,25 D_{3}$ ) on antimycobacterial properties of AMs, i.e. inhibition of intracellular Mtb growth and the production of immunoregulatory cytokines involved in TB immunity. Our hypothesis was that AMs of healthy humans would restrict Mtb growth in vitro better than AMs of individuals with a history of TB (previous or current), and that $1,25 \mathrm{D}_{3}$ stimulation in vitro would reinforce the host control of TB exercised by the infected AMs.

\section{Patients and methods}

\section{Study population}

Patients, planned for diagnostic bronchoscopy on the suspicion of active TB at the Department of Pulmonary Medicine, Linköping University Hospital, were consecutively included in the study during a 2-yrs-period after informed and written consent. Only patients that were not diagnosed by positive sputum smear for acid fast rods were included. Non-TB subjects were patients investigated by bronchoscopy because of a history of persistent cough. The purpose of bronchoscopy was in the case of the non-TB patient inspection mainly, since there was no suspicion of serious lung disease. The study protocol was approved by the Ethical Committee of Linköping University, Sweden. Patients were classified by a senior pulmonary physician and a senior thoracic radiologist as "non-TB", "previous TB" or "current TB" according to published guidelines [10]. The classification was performed without knowledge of the experimental results. Altogether, 24 patients were included; 8 healthy non-TB subjects (C1-8), 8 patients with previous TB (PTB1-8) and 8 patients with active TB (TB1-8). “Non-TB" were patients with negative TB-history/ PPD/IGRA-test, normal chest X-ray and/or high resolution computer tomography (HRCT), and bronchoscopy with negative mycobacterial cultures (4 patients were eligible; $C 2$, C3, C6, C8). "Previous TB" were patients with a medical history of culture-positive TB and radiological findings on chest X-ray and/or HRCT consistent with previous TB, supported by a positive TST and/or IGRA-test but negative cytology and negative mycobacterial cultures/PCR by bronchoscopy (4 patients were eligible; PTB1, PTB2, PTB4, PTB6). "Current TB" were patients that after bronchoscopy were treated for TB because of a positive mycobacterial culture and/or clinical and radiological findings suggestive of active TB ( 3 patients were eligible; TB2, TB3, TB8). The main reason behind noneligibility was problems associated with cell culturing, such as inadequate number of cells and unexpected cell death, which caused a lack of laboratory data in these cases. None of the patients investigated was treated with TB drugs at the time when blood samples and BALF were collected. All TBpatients included were HIV-negative, while the healthy nonTB-subjects were not tested for HIV. The patient characteristics are presented in (Table 1).

\section{Preparation of alveolar macrophages}

The BALF was taken care of immediately and centrifuged. The cells were counted, re-suspended in Dulbecco's modified Eagle's medium (DMEM; Gibco) supplemented with 10 $\%$ normal human serum, $100 \mathrm{U} / \mathrm{ml}$ penicillin, $100 \mu \mathrm{g} / \mathrm{ml}$ streptomycin (complete DMEM), and seeded in 96-well plates (Sarstedt; 100000 cells/well). Attached AMs were cultured for 5-7 days and the medium was changed to antibiotic-free DMEM at least one day prior to infection.

\section{Bacteria}

For details about the properties of the bacteria used see previous description [11,12]. Briefly, the virulent Mtb strain H37Rv (American Type Culture Collection) harbouring a pSMT1-plasmid encoding Vibrio harveyi luciferase were grown in Middlebrook $7 \mathrm{H} 9$ broth supplemented with Tween 80 and oleic acid-albumin-dextrose-catalase (OADC) (BD) for 2 to 3 weeks at $37^{\circ} \mathrm{C}$ with $100 \mu \mathrm{g} / \mathrm{ml}$ hygromycin for selection before being re-inoculated in fresh broth and incubated for 7 days to reach early log phase. Before infection, the bacterial suspension was washed and re-suspended in DMEM and a single-bacillus suspension was obtained by passage through a sterile syringe equipped with a 27 -gauge needle. The concentration was determined by optical density at 600 $\mathrm{nm}\left(\mathrm{OD}_{600}\right)$ as a function of colony-forming units $(\mathrm{CFU}) / \mathrm{ml}$.

\section{Infection procedure}

AMs were infected by a pulse-chase approach, where bacteria were added to the AMs in serum-free medium at a multiplicity of infection (MOI) of 10 and incubated at $37^{\circ} \mathrm{C}$ 
for $1 \mathrm{~h}$. The infection was followed by a change of medium to serum-containing antibiotic-free DMEM with $100 \mathrm{nM} \mathrm{1,25 \textrm {D } _ { 3 }}$ (Sigma-Aldrich). Control wells were given medium without added $1,25 \mathrm{D}_{3}$.

\section{Measurement of bacterial growth}

The number of viable bacteria was acquired through measurement of flash-luminescence with a GloMax ${ }^{\circledast}$ MultiDetection System (Promega) as previously described [13]. This method, based on flash-luminescence emitted from the luciferase-expressing bacteria, when given the luciferase substrate $n$-decanal, correlates well to CFUs, and display less variation than classic CFU assays. The phagocytic capacity of AMs [viable bacteria correspond to the flash-luminescence expressed as arbitrary luminescence units, (ALU)] was assessed at $1 \mathrm{~h}$ after infection. The capacity of AMs to control Mtb growth was assessed at 2 days of infection (expressed as fold change day 2 compared to day 0 ).

\section{Measurement of cytokine release}

The concentrations of cytokines (TNF- $\alpha$, IL-1 $\beta$, IL-10 and IL12p40), produced by Mtb-infected AMs, were quantified by a $\mathrm{BD}^{\mathrm{TM}}$ Cytometric Bead Array Flex Set (BD) according to the manufacturer's instructions. Thus, culture supernatants from infected AMs were collected at $24 \mathrm{hrs}$ post-infection, aliquoted and kept at $-70^{\circ} \mathrm{C}$. For analysis, samples were centrifuged at $10000 \times g$ for $10 \mathrm{~min}$ prior to dilution and mixing with cytokine-specific beads. The supernatant-bead suspensions were incubated for $1 \mathrm{~h}$ at room temperature, followed by $2 \mathrm{~h}$ of incubation in the presence of cytokine-specific PEconjugated detection antibodies. Samples were sterilized by adding 4\% paraformaldehyde (PFA) for $1 \mathrm{~h}$ before the final wash step. Flow cytometry (Gallios Flow Cytometer, Beckman Coulter) was used to quantitate each cytokine. Thus, each bead population was separated by a unique ratio of APC and APC-Cy7 and the fluorescence intensity of PE corresponded to the concentration of the specific cytokine, derived from a standard curve of known cytokine concentrations.

\section{Quantiferon assay}

Blood samples were collected in tubes provided by the manufacturer for the QuantiFERON ${ }^{\circledast}$-TB Gold In-Tube assay (Cellestis, Australia).Samples were shaken and incubated at $37^{\circ} \mathrm{C}$ overnight and then analyzed by ELISA for IFN- $\gamma$ (IU/ml) according to the manufacturer's instructions.

\section{Measurement of 25-hydroxyvitamin D in serum}

Using ELISA (Immmundiagnostik, Germany) according to the manufacturer's instructions, we measured the major circulating metabolite of vitamin $D, 25$-hydroxyvitamin $D$ (serum-25(OH)D).

\section{Statistics}

To evaluate the effects of $1,25 D_{3}$ stimulation within each group and comparison of the total non-stimulated with stimulated samples, a paired Student's t-test was used. The means between different study groups were compared using ANOVA with Bonferroni's post-hoc test. Differences between groups are shown as ${ }^{*}(p<0.05)$ or ${ }^{* *}(p<0.01)$ or ${ }^{* * *}(p<0.001)$.

\section{Results}

All eligible patients were thoroughly characterized and as shown in (Table 1), clinical symptoms, radiological findings as well as laboratory results rendered the classification into "non-TB", " previous TB" and "current TB". Patients with "current $\mathrm{TB}^{\prime}$ " tended to be younger, slimmer (lower BMI) and deficient of vitamin D (assessed with serum-25(OH)D). Their BALF contained more inflammatory cells, but compared to the other groups there were no statistical differences which was most likely because of few subjects. In one case of "previous TB", radiologic findings suggested "active $\mathrm{TB}^{\mathrm{B}}$ ", but bronchoscopy including mycobacterial culture and clinic-radiologic followup ruled out this possibility.

AMs isolated from patients with previous TB differed from those harvested from "non-TB" and "current TB" patients, when challenged with virulent Mtb. AMs isolated from "previous TB" patients exhibited a tendency towards a reduced phagocytic capacity of Mtb (Figure 1A,1C) and, importantly, significantly reduced efficiency to restrict Mtb growth ( $p<0.05$ vs. non-TB; (Figure 1B,1D). AMs harvested from patients with "previous" or "current TB" were clearly inferior to "non-TB" AMs to control Mtb infection in vitro (Figure 1B,1D).

To further investigate factors that may explain the variable capacity to control intracellular growth in AMs, we focused on vitamin $D$ stimulation in vitro and production of cytokines linked to innate immunity. No significant effects of $1,25 D_{3}$ stimulation of AMs on Mtb growth were observed (Figure 1E). Most cytokines were not detectable in the supernatants. Indeed, measurable levels were observed only for TNF- $a$, which remained at the same level when Mtbinfected AMs were stimulated with $1,25 D_{3}$ (data not shown).

\section{Discussion}

Experimental data on mycobacterial killing in freshly harvested BALF macrophages from patients with active or previous exposure to TB is scarce. In that perspective, we believe the results of our study is of importance, although it is small pilot study. As BALF is routinely collected in many centers, BALF studies may, indeed, become an excellent way to describe cellular effects of host defense to Mtb. Therefore, and despite the weaknesses of the present studies, we believe that the observation that AMs isolated from "previous TB" patients are less able to restrict Mtb growth, when challenged with virulent $\mathrm{Mtb}$, have opened a window towards a better understanding how the immune system operates to restrict Mtb growth. This observation indicates that the status (genotypic or phenotypic) of AMs could influence the ability of Mtb to establish infection, which supports the epidemiological observation that not 

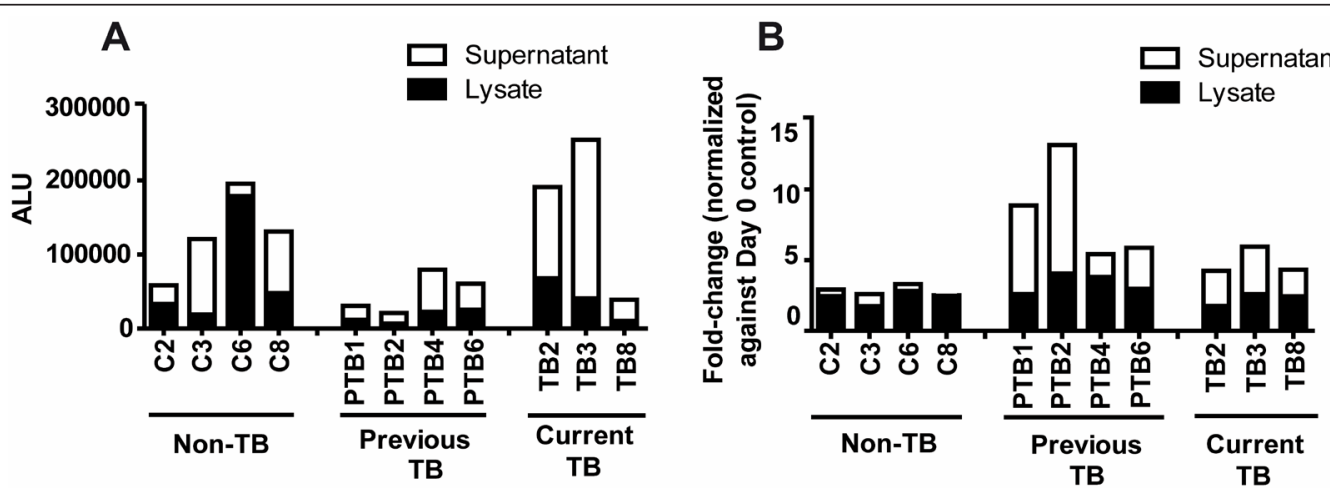

C

D
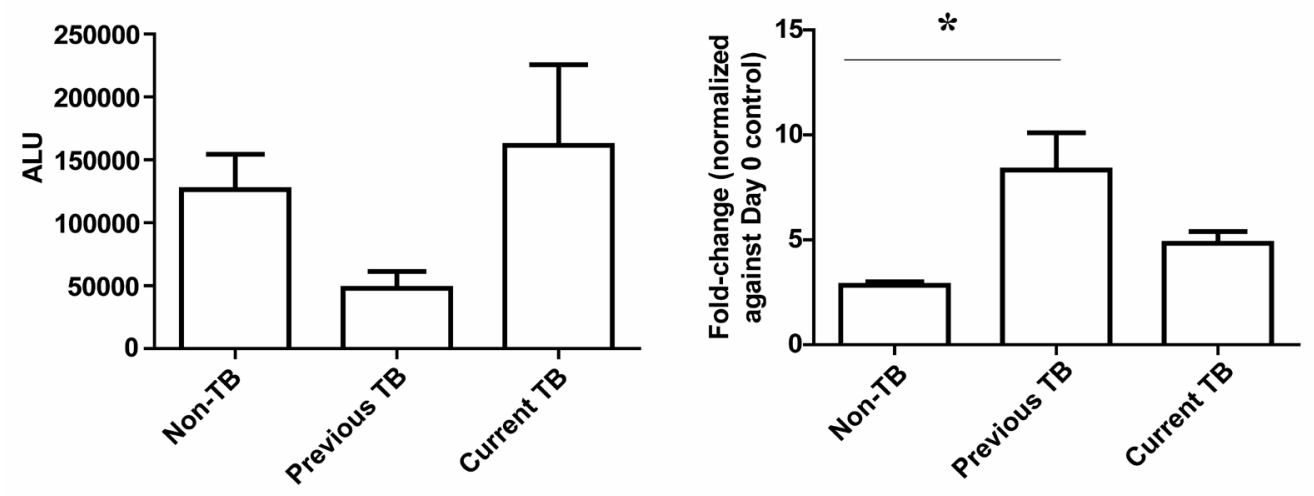

E

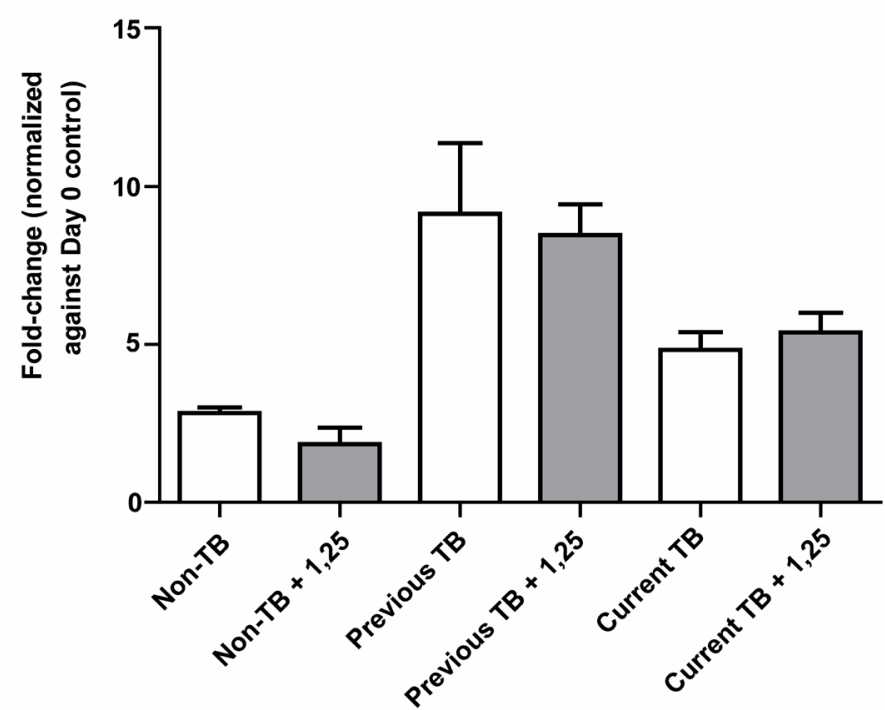

Figure 1. Phagocytosis and growth of H37Rv in cultures of BAL-macrophages.

Alveolar macrophages from the three groups of patients were infected with H37Rv at MOI 10 as described. Luminescence (ALU) was measured $1 \mathrm{~h}$ after pulse-chase (D0 value) as a measure of phagocytosis. After another 2 days of incubation, luminescence was measured again and growth was evaluated (D2 value). (A) The figure shows the ALU values from individual patients in each group with the proportion of lysate (i.e., bacteria inside AMs)- and supernatant (extracellular bacteria)associated bacteria $1 \mathrm{~h}$ after pulse-chase. (B) The figure shows the relative growth of H37Rv (D2/D0) in the cells of individual patients, with the proportion of relative growth in the supernatant (extracellular) and lysate (intracellular). (C) The mean ALU (intracellular + extracellular) value of each of the groups $1 \mathrm{~h}$ after pulse-chase (phagocytosis). (D) The mean relative growth (intracellular + extracellular) at 2 days post-infection from each group is shown. (E) The relative growth (intracellular + extracellular) of $\mathrm{H} 37 \mathrm{Rv}(\mathrm{D} 2 / \mathrm{D} 0)$ in the three study groups, in the absence or presence of 1,25-dihydroxyvitamin D3. Non-TB $(n=4)$, previous TB $(n=4)$ and current TB $(n=3)$. Bar graphs show the mean and SEM. The means were compared using ANOVA with Bonferroni's post-hoc test. Differences in growth with or without 1,25-dihydroxyvitamin D3 stimulation within each group was tested by a paired Student's t-test. 
more than $25-50 \%$ of close household contacts exposed to active pulmonary TB will acquire Mtb infection measured as a positive TST $[3,14]$. Moreover, our finding is well in line with a previous study showing that AMs from household contacts of patients with active TB are less capable of controlling Mtb infection compared to community controls [15]. In vivo, it should also be considered that other inflammatory cells may interact with AMs in the human lung during the innate immune response to hamper the growth of Mtb.

TB is a rare infection in Sweden. Most lung TB patients present with productive cough, and TB diagnosis is therefore often easily made by diagnostic tests for TB performed on sputum. Consequently, there is usually no medical reason to perform bronchoscopy. Hence, all TB-patients in the present study had negative TB-diagnostics of sputum, and most of them few symptoms of TB (Table 1). The main reason for bronchoscopy was radiologic findings indicative of TB infection, and the procedure was therefore performed either to rule out (patients later categorized as "previous TB") or to confirm a current infection (patients later categorized as "current TB"). Studies on BALF-cells are also associated with problems related to cell culturing, of which inadequate number of cells and unexpected cell death are the main issues to handle. Indeed, study patients underwent bronchoscopy to collect BALF for TB-diagnostics, and, subsequently, the portion of BALF decided for research sometimes was too small. Despite these major issues, altogether 11 of 24 cases included during a 2-yrs-period were considered eligible.

A limitation of the present study is the few study subjects in each group resulting in a potentially heterogeneous study population. Thus, a mismatch regarding important variables of the individuals included (sex, age, ethnicity, BMI, smoking habits and the level of vitamin $D$ in blood) may have some influence on the results of the present study. Notably, there was a trend for lower levels of serum-25(OH)D in patients with active TB, but there were too few subjects to make any firm conclusions. Since UVB-light is the main source to active vitamin $D$, the levels of serum-25(OH)D could show significant seasonal variations in countries, which like Sweden is situated close to the Poles. Although study subjects were included at all four seasons, clearly, all TB-subjects were deficient of vitamin $D$ (defined as serum-25(OH)D $\leq 35 \mathrm{nmol} / \mathrm{L}$ ) [16].

The presence of vitamin $D$ had no influence on Mtb growth in AMs, which is the opposite compared to results from $1,25 D_{3}$-stimulated human monocyte-derived macrophages (hMDM) which has been reported by us [17] and others $[4,5]$. However, it should be noted that monocyte-derived macrophages may be phenotypically different from AMs and are also differentiated in vitro. Thus, a different response to vitamin $\mathrm{D}$ between these two cell types is reasonable since other important differences such as that production of iNOS is present in AMs but not detectable in hMDMs have been reported [18]. Recently, we reported that $1,25 \mathrm{D}_{3}$-stimulation of hMDM, collected from the same individuals as in the present study, results in a hampered Mtb growth in infected cells as well as an increased production ofpro-inflammatory cytokines [17]. The lack of cytokine production following vitamin $D$ stimulation observed in the present study might therefore suggest that the reason behind AMs not responding to $1,25 \mathrm{D}_{3}$ would be on the phenotypic level. Alternatively, the lack of $1,25 \mathrm{D}_{3}$ influence might be explained by a rather short period for intra-cellular mycobacterial growth (2 days post-infection). This time-point for evaluation was based on pilot studies by our group showing that beyond 2 days of post-infection, less than $10 \%$ of monocyte-derived human macrophages are viable, when infected at an $\mathrm{MOI}$ of 10 [19]. In future studies, it might be of interest to extend the incubation time to better evaluate differences between $1,25 \mathrm{D}_{3}$ stimulated and nonstimulated cells in respect to bacterial growth.

\section{Conclusions}

In conclusion, the present study indicates that AMs retrieved from patients with a history of TB have a significantly reduced ability to control Mtb infection in vitro compared to AMs harvested from "non-TB" patients. This finding supports the concept that innate immunity is crucial for the control of infection. Future studies to verify this observation and evaluate the influence of vitamin D on human AMs are warranted.

\section{List of abbreviations}

AMs: alveolar macrophages

ALU: arbitrary luminescence units

BALF: bronchoalveolar lavage fluid

CFU: colony-forming units

$1,25 D_{3}, 1,25$-dihydroxyvitamin D

DMEM: Dulbecco's modified Eagle's medium

HRCT: high resolution computer tomography

hMDM: human monocyte-derived macrophages

Mtb: Mycobacterium tuberculosis

MOI: multiplicity of infection

OADC: oleic acid-albumin-dextrose-catalase

OD: optical density

PFA: paraformaldehyde

TB: tuberculosis

\section{Competing interests}

The authors declare that they have no competing interests.

\section{Authors' contributions}

All listed authors meet the criteria for authorship outlined by the International Committee for Medical Journal Editors.

\section{Acknowledgement and funding}

The authors acknowledge the invaluable contribution of the Bronchoscopy Unit and the nursing staff (Ms. P. Jacobson in particular) at the Dept. of Pulmonary Medicine, Linköping, Sweden. This work was supported by grants from the medical research council of south east Sweden (FORSS).

\section{Publication history}

Received: 14-Feb-2013 Revised: 07-Apr-2013

Accepted: 12-Apr-2013 Published: 15-Apr-2013

\section{References}

1. Natarajan K, Kundu M, Sharma P and Basu J: Innate immune responses 
Lennart et al. Microbiology Discovery 2013,

to M. tuberculosis infection. Tuberculosis (Edinb) 2011, 91:427-31. | Article | PubMed

2. Cooper AM: Cell-mediated immune responses in tuberculosis. Annu Rev Immunol 2009, 27:393-422. | Article | PubMed

3. Morrison J, Pai M and Hopewell PC: Tuberculosis and latent tuberculosis infection in close contacts of people with pulmonary tuberculosis in low-income and middle-income countries: a systematic review and meta-analysis. Lancet Infect Dis 2008, 8:359-68. | Article | PubMed

4. Crowle AJ, Ross EJ and May MH: Inhibition by 1,25(OH)2-vitamin D3 of the multiplication of virulent tubercle bacilli in cultured human macrophages. Infect Immun 1987, 55:2945-50. | Article I PubMed Abstract | PubMed Full Text

5. Rook GA, Steele J, Fraher L, Barker S, Karmali R, O'Riordan J and Stanford $\mathrm{J}$ : Vitamin D3, gamma interferon, and control of proliferation of Mycobacterium tuberculosis by human monocytes. Immunology 1986, 57:159-63. | PubMed Abstract | PubMed Full Text

6. Liu PT, Stenger S, Li H, Wenzel L, Tan BH, Krutzik SR, Ochoa MT, Schauber J, Wu K, Meinken C, Kamen DL, Wagner M, Bals R, Steinmeyer A, Zugel U, Gallo RL, Eisenberg D, Hewison M, Hollis BW, Adams JS, Bloom BR and Modlin RL: Toll-like receptor triggering of a vitamin D-mediated human antimicrobial response. Science 2006, 311:1770-3. | Article I PubMed

7. Larcombe L, Orr P, Turner-Brannen E, Slivinski CR, Nickerson PW and Mookherjee N: Effect of vitamin D supplementation on Mycobacterium tuberculosis-induced innate immune responses in a Canadian Dene First Nations cohort. PLoS One 2012, 7:e40692. | Article | PubMed Abstract | PubMed Full Text

8. Wejse C, Gomes VF, Rabna P, Gustafson P, Aaby P, Lisse IM, Andersen PL, Glerup $H$ and Sodemann $M$ : Vitamin $D$ as supplementary treatment for tuberculosis: a double-blind, randomized, placebo-controlled trial. Am J Respir Crit Care Med 2009, 179:843-50. I Article I PubMed

9. Martineau AR, Timms PM, Bothamley GH, Hanifa Y, Islam K, Claxton AP, Packe GE, Moore-Gillon JC, Darmalingam M, Davidson RN, Milburn HJ, Baker LV, Barker RD, Woodward NJ, Venton TR, Barnes KE, Mullett CJ, Coussens AK, Rutterford CM, Mein CA, Davies GR, Wilkinson RJ, Nikolayevskyy V, Drobniewski FA, Eldridge SM and Griffiths CJ: Highdose vitamin $\mathrm{D}(3)$ during intensive-phase antimicrobial treatment of pulmonary tuberculosis: a double-blind randomised controlled trial. Lancet 2011, 377:242-50. | Article | PubMed

10. Diagnostic Standards and Classification of Tuberculosis in Adults and Children. This official statement of the American Thoracic Society and the Centers for Disease Control and Prevention was adopted by the ATS Board of Directors, July 1999. This statement was endorsed by the Council of the Infectious Disease Society of America, September 1999. Am J Respir Crit Care Med 2000, 161:1376-95. I PubMed

11. Eklund D, Welin A, Schon T, Stendahl O, Huygen K and Lerm M: Validation of a medium-throughput method for evaluation of intracellular growth of Mycobacterium tuberculosis. Clin Vaccine Immunol 2010, 17:513-7. I Article | PubMed Abstract | PubMed Full Text

12. Welin A, Raffetseder J, Eklund D, Stendahl O and Lerm M: Importance of phagosomal functionality for growth restriction of Mycobacterium tuberculosis in primary human macrophages. J Innate Immun 2011, 3:508-18. I Article I PubMed

13. Eklund D, Welin A, Schon T, Stendahl O, Huygen K and Lerm M: Validation of a medium-throughput method for evaluation of intracellular growth of Mycobacterium tuberculosis. Clin Vaccine Immunol 2010, 17:513-7. | Article | PubMed Abstract | PubMed Full Text

14. Grzybowski S, Barnett GD and Styblo K: Contacts of cases of active pulmonary tuberculosis. Bull Int Union Tuberc 1975, 50:90-106. | PubMed

15. Carranza C, Juarez E, Torres M, Ellner JJ, Sada E and Schwander SK: Mycobacterium tuberculosis growth control by lung macrophages and CD8 cells from patient contacts. Am J Respir Crit Care Med 2006, 173:238-45. | Article | PubMed Abstract | PubMed Full Text

16. Ross AC: The 2011 report on dietary reference intakes for calcium and vitamin D. Public Health Nutr 2011, 14:938-9. | Article | PubMed

17. Eklund D, Persson HL, Larsson M, Welin A, Idh J, Paues J, Fransson SG, Stendahl O, Schön T, Lerm M: Vitamin D enhances IL-1b secretion and restricts growth of Mycobacterium tuberculosis inmacrophages from TB patients. Int. J. Mycobacteriol 2013, 2:18-25. I Article

18. Nathan $\mathrm{C}$ and Shiloh MU: Reactive oxygen and nitrogen intermediates in the relationship between mammalian hosts and microbial pathogens. Proc Natl Acad Sci U S A 2000, 97:8841-8. | Article | PubMed Abstract | PubMed Full Text

19. Welin A, Eklund D, Stendahl O and Lerm M: Human macrophages infected with a high burden of ESAT-6-expressing $M$. tuberculosis undergo caspase-1- and cathepsin B-independent necrosis. PLOS One 2011, 6:e20302. | Article | PubMed Abstract | PubMed Full Text

\section{Citation:}

Lennart P H, Eklund D, Larsson M, Welin A, Paues J, Idh J, Fransson S G, Stendahl O, Lerm M and Schon T: Alveolar macrophages from patients with tuberculosis exhibit reduced capacity of restricting growth of Mycobacterium tuberculosis: a pilot study of vitamin D stimulation in vitro. Microbiology Discovery 2013, 1:6. http://dx.doi.org/10.7243/2052-6180-1-6 